\title{
GALACTIC DYNAMOS - A CHALLENGE FOR OBSERVERS
}

\author{
RAINER BECK \\ Max-Planck-Institut für Radioastronomie, Auf dem Hügel 69, W-5300 Bonn 1, FRG
}

January 13, 1993

\begin{abstract}
Results of linear $\alpha \Omega$-dynamo models are confronted with radio polarization observations of spiral galaxies. The general distribution of polarized emission and the magnetic field pitch angle can be described with sufficient accuracy. The occurrance of systematic large-scale variations in Faraday rotation $(R M)$ is the strongest argument in favour of dynamo theory. However, the predominance of axisymmetric SO modes could not be confirmed by observations; S1 modes are about equally frequent. The azimuthal variations of field pitch angles and, in two cases, the phases of the $R M$ variations are inconsistent with a classical $\alpha \Omega$-dynamo. Locally deviating $R M$ values indicate field lines bending out of the plane. There is increasing evidence that galactic fields cannot be described by simple dynamo modes. This calls for more realistic dynamo models, taking into account non-axisymmetric velocity fields and galactic winds.

Interpretation of radio observations is difficult because Faraday depolarization can seriously affect the data. Observations of small-scale field structures are summarized which show the path for future research. Instrumental needs for such investigations are discussed.
\end{abstract}

\section{Historical Dates}

The observations of interstellar magnetic fields produce major discoveries roughly every ten years. 60 years ago Jansky published his first observations of cosmic synchrotron radiation. 50 years ago Öhman reported the detection of optical polarization in the Andromeda Galaxy (M31) and opened another way to study interstellar magnetic fields. 30 years ago, Westerhout and Wielebinski independently discovered polarized radio emission from the Milky Way, bringing the technique of radio polarization into the field (see also Wielebinski, this volume). Polarized radio emission from an external spiral galaxy (M51) was first reported by Mathewson 20 years ago, the same year as the inauguration of the Effelsberg 100-m telescope. Based on a detailed polarization survey of M31 with this instrument, the relevance of the uni-directional field in M31 as an indication of a large-scale galactic dynamo was first mentioned by the author -10 years ago. Hence a cumulation of anniversaries occurs in 1992, strong arguments for a review of the present observational data confronted with the theory of galactic dynamos, asking:

Do galactic dynamos exist?

\section{Why Testing Dynamo Theory in Galaxies?}

- Galaxies are transparent to unpolarized radio waves at decimeter and centimeter wavelengths, and transparent for polarized radio waves at centimeter wavelengths. Hence the 3-D structure of their magnetic fields can be measured, in contrast to opaque bodies like planets and stars.

- The line-of-sight component of the gas velocity field can be measured, yielding information about the differential rotation and its spatial variation. Highresolution velocity data can (in principle) provide the $\mathbf{v} \times \mathbf{B}$ term essential for dynamo action.

- Radio polarization data provide detailed data about the magnetic field structure. 
TABLE I

Magnetic field structures of normal galaxies as derived from radio polarization data

\begin{tabular}{|c|c|c|}
\hline Galaxy & Wavelength & Field structure \\
\hline M31 & $\begin{array}{l}\text { E } 11,6 \mathrm{~cm} \\
\mathrm{~V} 20 \mathrm{~cm}\end{array}$ & $\underline{\text { ASS }}(+$ BSS?) \\
\hline M33 & E $21,18,11,6,2.8 \mathrm{~cm}$ & Spiral, BSS? \\
\hline M51 & $\begin{array}{l}\text { E } 6,2.8 \mathrm{~cm} \\
\text { W } 21 \mathrm{~cm} \\
\text { V } 20,18,6 \mathrm{~cm}\end{array}$ & Spiral, ASS+BSS? \\
\hline M81 & $\begin{array}{l}\mathrm{E} \quad 6 \mathrm{~cm} \\
\mathrm{~W} \quad 21 \mathrm{~cm} \\
\mathrm{~V} \quad 20,6 \mathrm{~cm}\end{array}$ & $\underline{\text { BSS }}$ \\
\hline M83 & $\begin{array}{l}\mathrm{E} \quad 6,2.8 \mathrm{~cm} \\
\mathrm{~V} 20,6 \mathrm{~cm}\end{array}$ & Bisymmetric and along bar \\
\hline M101 & E $6 \mathrm{~cm}$ & Spiral \\
\hline M104 & $\mathrm{V} 20,6 \mathrm{~cm}$ & $?$ \\
\hline SMC & P $21,12 \mathrm{~cm}$ & $?$ \\
\hline LMC & P $21,12,6 \mathrm{~cm}$ & Loop south of 30 Dor \\
\hline IC 342 & $\begin{array}{l}\mathrm{E} 11,6 \mathrm{~cm} \\
\mathrm{~V} 20,6,3.5 \mathrm{~cm}\end{array}$ & $\underline{\mathrm{ASS}}$ \\
\hline NGC 877 & $\mathrm{~V} 20,6 \mathrm{~cm}$ & $?$ \\
\hline NGC 1566 & A $6 \mathrm{~cm}$ & Spiral \\
\hline NGC 1672 & A $6 \mathrm{~cm}$ & Spiral \\
\hline NGC 1808 & $\mathrm{~V} 20,6 \mathrm{~cm}$ & Extensions $\perp$ plane \\
\hline NGC 2207 & $\mathrm{~V} 20,6,3.6 \mathrm{~cm}$ & Along spiral arm segment \\
\hline NGC 2276 & $\mathrm{~V} 20,6 \mathrm{~cm}$ & Spiral \\
\hline NGC 2903 & $\begin{array}{l}\text { E } 6,2.8 \mathrm{~cm} \\
\text { V } 18,20 \mathrm{~cm}\end{array}$ & Spiral \\
\hline NGC 4038/39 & $\mathrm{V} 20,6,3.6 \mathrm{~cm}$ & Along tidal arm \\
\hline NGC 4258 & $\begin{array}{l}\text { E } 6,2.8 \mathrm{~cm} \\
\text { W } 49,21 \mathrm{~cm} \\
\text { V } 20,6 \mathrm{~cm}\end{array}$ & Along anomalous arms \\
\hline NGC 6946 & $\begin{array}{l}\mathrm{E} 6,2.8 \mathrm{~cm} \\
\mathrm{~W} 21 \mathrm{~cm} \\
\mathrm{~V} 20,18,6 \mathrm{~cm}\end{array}$ & Spiral, ASS? \\
\hline
\end{tabular}

ASS: Axisymmetric spiral structure (dynamo mode S0)

BSS: Bisymmetric spiral structure (dynamo mode S1)

$\begin{array}{lll}\text { Instruments: } & \mathrm{E}=\text { Effelsberg } 100-\mathrm{m} & \mathrm{A}=\text { Australia Telescope Compact Array } \\ & \mathbf{P}=\text { Parkes } 64-\mathrm{m} & \mathrm{W}=\text { Westerbork Synthesis Radio Telescope } \\ \mathrm{V}=\text { Very Large Array } & \end{array}$ 
TABLE I (contd.)

\begin{tabular}{lll}
\hline Galaxy & Wavelength & Field structure \\
\hline Edge - on galaxies : & & \\
\hline NGC 253 & E $2.8 \mathrm{~cm}$ & $\|$ plane \\
& P $6 \mathrm{~cm}$ & \\
& V $20,6,3.5 \mathrm{~cm}$ & \\
NGC 891 & V $20,6 \mathrm{~cm}$ & $\|$ and inclined to plane \\
& E $2.8 \mathrm{~cm}$ & \\
NGC 3628 & E $2.8 \mathrm{~cm}$ & $\|$ and inclined to plane \\
& V $20,6 \mathrm{~cm}$ & \\
NGC 4565 & V $20,6 \mathrm{~cm}$ & $\|$ plane \\
NGC 4631 & E $6,2.8 \mathrm{~cm}$ & $\perp$ plane (inner region), \\
& V $20,6,3.5 \mathrm{~cm}$ & $\|$ and inclined to plane (outer regions) \\
NGC 4945 & P $6 \mathrm{~cm}$ & Extensions $\perp$ plane \\
& A $20,13 \mathrm{~cm}$ & \\
NGC 5775 & V $6 \mathrm{~cm}$ & $\|$ plane \\
NGC 7331 & V $20,6 \mathrm{~cm}$ & $?$ \\
& E $2.8 \mathrm{~cm}$ & \\
\hline
\end{tabular}

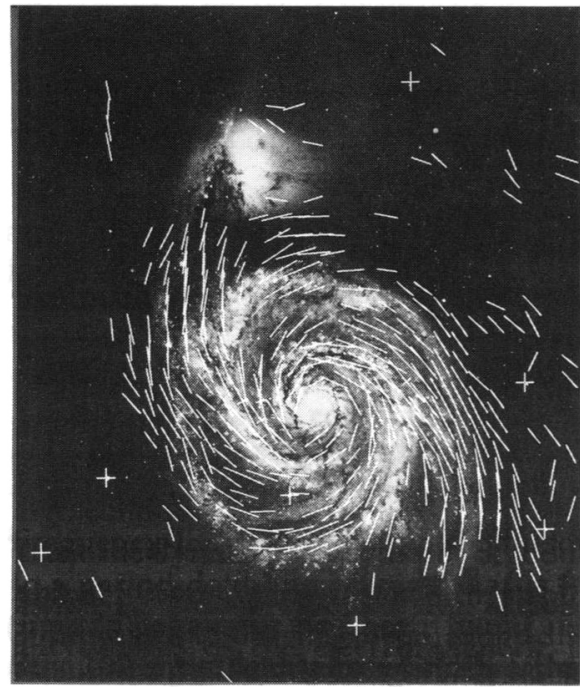

Fig. 1. Magnetic field orientations (Bvectors) in M51, derived from Effelsberg observations at $\lambda 2.8 \mathrm{~cm}$ with $72^{\prime \prime}$ resolution (Neininger 1992a; see also Neininger et al., this volume).

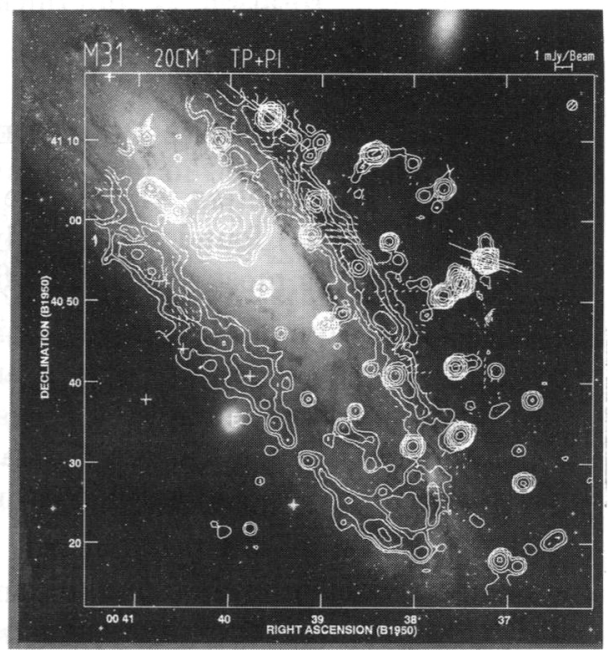

Fig. 2. Total and polarized emission from the southern half of M31 at $\lambda 20.1 \mathrm{~cm}$, observed with the VLA in its D configuration (75" synthesized beam). E-vectors (shown here) are uncorrected for Faraday rotation (Beck, Berkhuijsen and Hummel, unpublished). 


\section{Radio Polarization Observations}

Linearly polarized radio waves carry information about the strength and orientation of the magnetic field component $B_{\perp}$ perpendicular to the line of sight, averaged along the line of sight and within the telescope beam. At short wavelengths (typically $\leqq 6 \mathrm{~cm}$ ) the $\mathbf{E}$-vectors are nearly perpendicular to $B_{\perp}$; at longer wavelength they are rotated proportional to $B_{\|}$along the line of sight. Observational techniques are described in detail by M. Krause (1990) and Beck (1991a) and discussed in Sect. 6. Table I summarizes the polarization data presently available.

\section{Classical Galactic Dynamo Models Confronted with Observations}

Elstner et al. (1992) provided the most extensive set of classical linear $\alpha \Omega$-dynamo models to be compared with radio polarization data. Their assumptions were:

- axial symmetry of gas distribution, gas velocity field and all model parameters

- simple rotation curve (rigid + differential)

$-\alpha$-effect mainly concentrated in the disk

- conductivity $\sigma$ decreases with $z$

- full thickness of synchrotron-emitting disk $=1 \mathrm{kpc}$

- full thickness of ionized gas disk $=1 \mathrm{kpc}$

From two basic parameters the fields were calculated:

$X=r_{\Omega} / z_{\alpha} \quad\left(r_{\Omega}\right.$ : turnover radius between rigid rotation $\Omega_{\max }$ and differential rotation; $z_{\alpha}$ : full thickness of the disk where the $\alpha$-effect operates) $C_{\Omega}=\mu \sigma \Omega_{\max } z_{\alpha}^{2}$ (dynamo number)

\subsection{Distribution of the Polarized Radio Intensity}

For all model parameters which were thought to be realistic for spiral galaxies Elstner et al. (1992) found that the dynamo mode SO is mostly excited first and should dominate in most galaxies. S0 fields can be characterized by:

- a central minimum of polarized emission,

- a maximum polarized intensity near $r_{\Omega}$,

- a ring of polarized emission with its width increasing with decreasing $X$,

- edge-on view: a thick disk of polarized radio emission with its thickness increasing with decreasing dynamo number $C_{\Omega}$, and the orientation of B-vectors parallel to the disk.

These properties agree with radio maps (Fig. 3) if $X$ lies between 1 (NGC 6946) and 5 (M31), i.e. the $\alpha$-effect operates until a large height above the plane. This seems to rule out an $\alpha$-effect driven by supernova explosions in the plane. The lack of central polarized emission in the model can be removed by dynamo-driven accretion (Rüdiger et al. 1993) or by a more realistic rotation curve.

Edge-on galaxies like NGC 891 (thick radio disk) and NGC 4565 (thin radio disk, see Sukumar \& Allen 1991) can also be described by S0 fields with different values of $X$ and $C_{\Omega}$ due to different rotation curves. Plane-parallel disk fields, in agreement with dynamo models, have been found in the edge-on galaxies NGC 3628 (Reuter et al. 1991) and NGC 4565 (Sukumar \& Allen 1991). NGC 891 seems to 
be an intermediate case where the field orientations are neither plane-parallel nor radial (Hummel et al. 1991; Sukumar \& Allen 1981) and may indicate a slow galactic wind (Brandenburg et al. 1993).

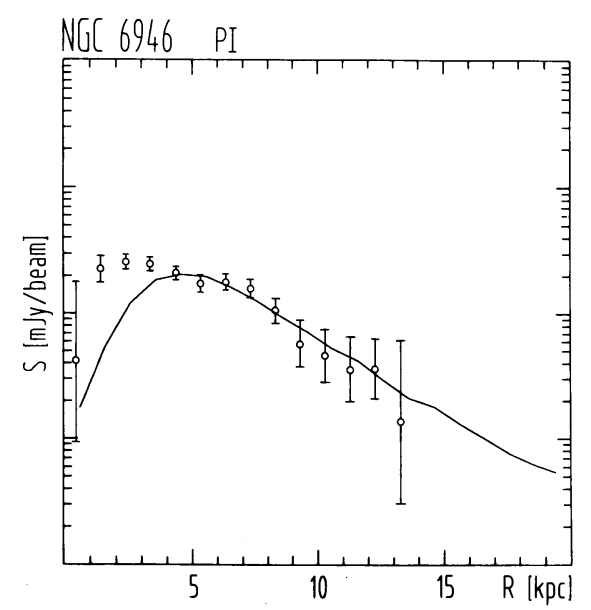

Fig. 3. Radial variation of the polarized emission from NGC 6946. The data points are $2.8 \mathrm{~cm}$ Effelsberg observations from Ehle \& Beck (1993), the solid line represents the dynamo model according to Elstner et al. (1992).

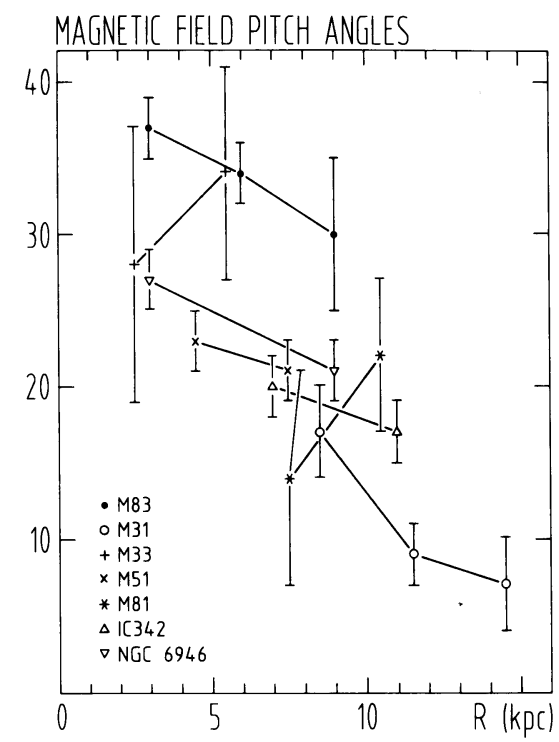

Fig. 4. Observed radial variation of field pitch angles

NGC 4631 with its huge radio halo and radial field in the inner region (Hummel et al. 1991; Golla \& Hummel, this volume) is in conflict with the predictions of classical dynamo theory. NGC 4631 resembles the dipole field of the antisymmetric A0 mode, possibly excited by the interaction with NGC 4656. Alternatively, NGC 4631 could be a case of the wind-driven dynamo without invoking any $\alpha$-effect (Spencer $\&$ Cram 1992), or a combination of a galactic wind and classical $\alpha \Omega$-dynamo (Brandenburg et al. 1993), or a wind-driven field without any dynamo (Lesch \& Harnett 1993).

Recently, another case of a huge synchrotron halo has been discovered with the VLA around NGC 253 (Carilli et al. 1992) and confirmed most recently with the Effelsberg telescope (Beck et al., in prep.). However, nothing is yet known about the field structure in this halo. The disk field is mainly parallel to the plane (Klein et al. 1983).

\subsection{Magnetic Field Pitch Angles}

Observations of moderately inclined galaxies show that the magnetic field closely follows the orientation of the optical spiral arms (Fig. 1) with average pitch angles of $10^{\circ}-35^{\circ}$ (Fig. 4). Pitch angles similar to the observed ones can be obtained in linear dynamo models (Elstner et al. 1992) with $C_{\Omega} \cong 10$. This is compatible with 
very small values for the conductivity (high turbulent resistivity), as assumed for the halo (Brandenburg et al. 1992), or very thin disks.

A coupling to the pitch angle of the optical spiral arms is impossible in axisymmetric dynamo models. The observed field pitch angle varies with azimuthal angle and seems to be influenced by gas streaming motions (Neininger 1992a,b). The pitch angle is smaller in interarm regions compared with spiral arms (Ehle \& Beck 1993). Hence an interaction between density waves and the dynamo should be considered (Chiba \& Tosa 1990; Hanasz et al. 1991; Chiba, this volume). Other non-axisymmetric gas velocities can also influence the magnetic field structure, e.g. around the bar of M83 (Neininger et al. 1991).

In symmetric (S-) modes the field pitch angle decreases with galactocentric distance (Chiba \& Tosa 1989; Krasheninnikova et al. 1989), while it increases for antisymmetric (A-) modes (Elstner et al. 1992). Observations (Fig. 4) indeed show a generally decreasing pitch angle. The only exceptions are M81 and possibly also M33, both candidates for S1-modes due to interaction (see Sect. 4.3).

\subsection{Faraday Rotation Measures}

The observations discussed until now provide necessary, but insufficient tests of dynamo theory, as other models of the origin of interstellar magnetic fields can provide similar properties. This changes if we consider Faraday rotation which shows the direction (not only the orientation) of the field. Dynamos per se generate magnetic fields which have the same direction over large distances. The preferred spiral field of type S0 either points inwards or outwards everywhere in a galaxy, seen from its center. Models favouring a local field origin can never obtain such a large-scale coordination of field directions.

Axisymmetric S0 fields can be detected by sinusoidal variations of Faraday rotation with azimuthal angle (at constant galactocentric radius) while bisymmetric S1 fields produce doubly-periodical $R M$ variations (e.g. Fig. 6). The pessimistic view of this method by Donner \& Brandenburg (1990) only refers to some fixed radius. If the radius of maximum polarized radio emission is considered, the sinusoidal variation is a good indicator of a S0 mode if the galaxy's inclination and disk thickness is not too large (Elstner et al. 1992). The phase of the variation is about equal to the pitch angle of the field at that radius. For large inclinations or thick disks (i.e. $X \cong 1$ ), the variation becomes asymmetric.

A0- and A1-modes also show singly-periodical variations of Faraday rotation, but their phases differ largely from the value of the pitch angle. Furthermore, A0and A1-modes have their maximum polarized emission in the inner regions of the galaxy, in contrast to S0 modes (Sect. 4.1).

In real galaxies, the variation of Faraday rotation will be disturbed by:

- non-axisymmetric distribution of field strength, cosmic rays or thermal gas,

- pitch angles varying with azimuthal angle,

- vertical field components,

- Faraday depth effects (see Sect. 6.4).

In spite of such obstacles, clear singly-periodical $R M$ variations could be detected in M31 (Beck 1982; Ruzmaikin et al. 1990), IC 342 (Gräve \& Beck 1988; M. Krause et 
al. 1989a; Sokoloff et al. 1992) and NGC 6946 (Ehle \& Beck 1993, and this volume). However, in NGC 6946 the phase largely differs from the mean pitch angle value: The $R M$ maximum and minimum lie on the wrong side of the galaxy's major axis (Fig. 5). Furthermore, the pitch angle varies with azimuthal angle. In M31 Sofue \& Beck (1987) found indications for a higher mode of lower amplitude superimposed onto the dominating $\mathrm{S} 0$ mode.

Fig. 5. Faraday rotation measures in NGC 6946, obtained from Effelsberg observations at $\lambda 6.3 \mathrm{~cm}$ and $\lambda 2.8 \mathrm{~cm}$. A foreground rotation measure of $36 \mathrm{rad} \mathrm{m}^{-2}$ has been subtracted (Ehle \& Beck 1993; see also Ehle \& Beck, this volume).

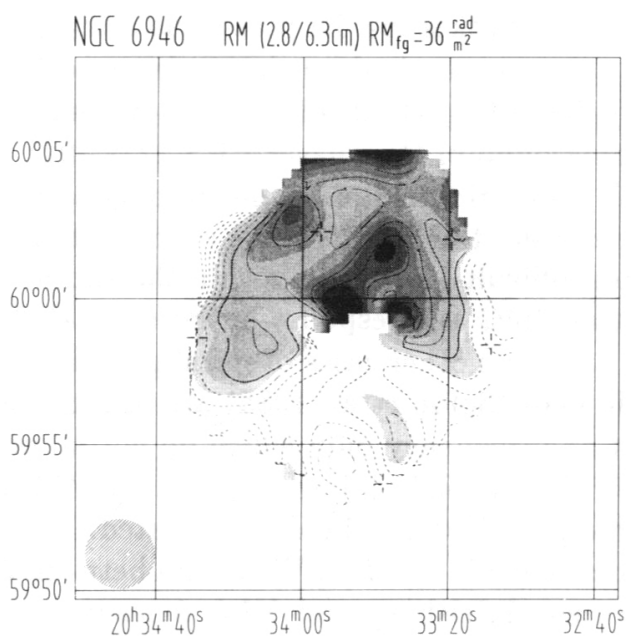

TABLE II

Large-scale structures of disk fields (revised)

\begin{tabular}{lll}
\hline Axisymmetric & Bisymmetric & Neither \\
\hline M31 & M81 & M51 \\
IC 342 & M33(?) & \\
NGC 6946(?) & M83(?) & \\
& PKS 1229-021(?) & \\
\hline
\end{tabular}

Two (and a half) cases - by far insufficient to confirm the dominance of S0 field structures as predicted by classical dynamo models. However, counterexamples are similarly rare (Table II).

Bisymmetric S1 fields are characterized by a doubly-sinusoidal variation of Faraday rotation. However, any doubly-periodical variation of the effects discussed above can mimic a S1 field. A true S1 variation has to fulfil a relation between its zerolevel, the field pitch angle and the foreground rotation (M. Krause et al. 1989b). Furthermore, the phase of a S1 variation has to change with galactocentric distance following the spiral shape.

The only clear candidate for housing a S1 field is M81 (M. Krause et al. 1989b; Sokoloff et al. 1992). Recent $\lambda 6 \mathrm{~cm}$ VLA data (Fig. 7) have confirmed a jump in $R M$ in the western half of the galaxy as expected for a bisymmetric field (Schoofs 
1992). The position of the jump (the magnetic neutral line) is no longer in the interarm region as suggested by $M$. Krause et al. (1989b), but near the outer edge of the inner spiral arm, consistent with the distribution of polarized emission.

In M33 the weak polarized emission leads to high uncertainties in Faraday rotation, and a bisymmetric field can be claimed only with some caution (Buczilowski \& Beck 1991). M33 and M81 show signs of gravitational interaction which can produce non-axisymmetric dynamo fields (Moss et al. 1993, and this volume). Vallée (1990) invokes low HI masses as a trigger for bisymmetric fields.

A new method to detect large-scale magnetic structures has been discussed by Kronberg et al. (1992). They found doubly-periodical $R M$ variations in the foreground of the quasar PKS 1229-021 which they interprete as being due to the bisymmetric field of an intervening spiral galaxy at $z=0.395$. As the $R M$ errors are large, this result is not conclusive. In any case, strong fields in young galaxies are difficult to explain within the timescales of current dynamo models (see Wolfe et al. 1992 and response by Perry et al. 1992).

Several other cases of spiral galaxies claimed to possess a dominating bisymmetric field (Sofue et al. 1985) are of much lower significance due to insufficient data at one frequency only which do not allow to compute Faraday rotation measures.

The strongly interacting galaxy M51 is a special case where the pitfalls of data interpretation can be demonstrated. It was previously thought to contain a bisymmetric field, based on VLA data between $\lambda 18 \mathrm{~cm}$ and $\lambda 20 \mathrm{~cm}$ (Horellou et al. 1990). Recent Effelsberg and VLA measurements at shorter wavelength do not show a clear variation of Faraday rotation (Neininger 1992b). The field structure seems to be disturbed by the interaction and cannot be described by a low-mode dynamo field. At longer wavelength Faraday depolarization becomes strong so that we may detect polarized emission only from the upper disk or halo (Horellou et al. 1992; Neininger et al., this volume). Some $R M$ structures correlate with the HI velocity field, possibly due to vertical fields. Variations in Faraday depth may mimic a doubly-periodical variation of Faraday rotation. Alternatively, the field in M51 can be described by an axisymmetric and a bisymmetric component with about equal weights (Shukurov, priv. comm.). The M51 field remains a mystery.

Low Faraday rotation in NGC 6946 and M83 led Beck (1991a) to introduce a third class of galaxies with a field structure of "neither" type. This may be the effect of Faraday depolarization (Sect. 6.4) - these galaxies are not transparent to polarized emission at decimeter wavelengths (Beck 1991b; Neininger et al. 1993). New observations at centimeter wavelengths exhibited much larger Faraday rotation. The field structure of M83 now seems to be bisymmetric (Fig. 6), but the phase of the $R M$ variations does not change with galactocentric distance. Such a field is incompatible with classical $\alpha \Omega$-dynamo models.

Doubly-periodical $R M$ variations with constant phase may indicate a superposition of a disk S0 field and a halo A0 field (Brandenburg et al. 1992). However, there is doubt that classical dynamo theory can be applied to the barred galaxy M83. Neither the gas distribution nor the velocity field is axisymmetric. Another type of dynamo, a "dynamical dynamo", may operate here. Gas streaming motions in the gravitational potential of the M83 bar are able to enhance magnetic fields without invoking the $\alpha$-effect (Lesch, this volume). 
Fig. 6. Azimuthal variation of Faraday rotation measures in two radius intervals in M83, obtained from Effelsberg observations at $\lambda 6.3 \mathrm{~cm}$ and $\lambda 2.8 \mathrm{~cm}$ (Neininger 1992b; Neininger et al. 1993).

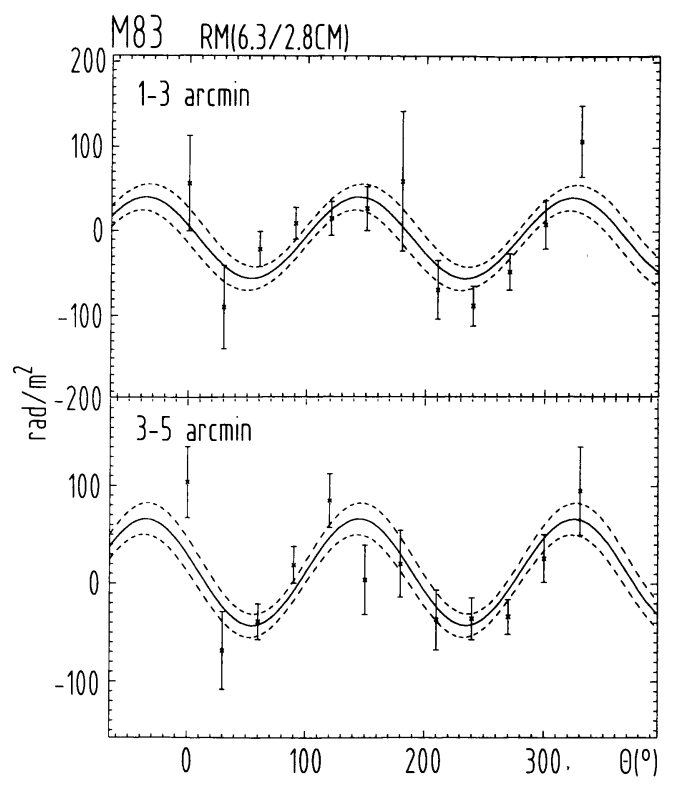

\subsection{Test Summary}

Mean-field dynamo theory cannot treat the small-scale structures of galactic magnetic fields. The predictions of classical $\alpha \Omega$-dynamo models are roughly consistent with large-scale field structures (Table III). The large number of exceptions demonstrates the limitations of present-day models. Fair agreement between theory and observations is found for just two galaxies: M31 and IC 342. In all other cases major discrepancies require modifications of dynamo models: Even the mean large-scale fields cannot be described by a simple symmetry mode. Special regards should be given to the gas motions within and out of the disk which may drive a dynamo

TABLE III

Testing mean-field $\alpha \Omega$-dynamo theory

\begin{tabular}{|l|l|}
\hline Prediction & Observation \\
\hline Uniform fields strongest near $r \Omega$ & Yes \\
Most uniform fields I plane & Yes (exception: NGC 4631) \\
No large radio halos & Yes (exceptions: NGC 253, NGC 4631) \\
Field pitch angle & Yes (exceptions: M81, M33(?)) \\
decreases with radius & Yes (exception: NGC 6946) \\
Phase of $R M$ variation of & \\
S0 fields $\cong$ pitch angle & Yes (exception: M83) \\
Phase of $R M$ variation of & \\
S1 fields changes with radius & \\
\hline
\end{tabular}


without help of helical turbulence ( $\alpha$-effect). Table IV summarizes the present-day problems with dynamo models.

\section{TABLE IV}

Limitations of present-day galactic dynamo models

\begin{tabular}{|l|l|}
\hline Problem & Examples \\
\hline Non-axisymmetric gas flow & M83 \\
Non-axisymmetric gas distribution & NGC 6946 \\
Gravitational interaction & M51, M81, NGC 4631 \\
Galactic wind & NGC 4631 \\
Vertical fields & M51, NGC 4631, NGC 6946 \\
Orientation of field lines parallel to optical spiral arms & general \\
Strongest uniform fields outside optical spiral arms & general \\
Field pitch angle varies with azimuthal angle & general \\
\hline
\end{tabular}

In spite of the discrepancies between dynamo models and several observational facts, the detection of large-scale patterns of Faraday rotation (e.g. Fig. 5) provides the most obvious hint for the action of a galactic dynamo.

\section{Small-scale Field Structures}

With increasing resolution of radio observations we now start to see magnetic field details which cannot be treated by mean-field dynamo theory. Similarities to the history of the observations of solar magnetic fields are obvious.

The almost toroidal S0 field of M31 shows loop structures with $\cong 4.5 \mathrm{kpc}$ "wavelength" (Beck et al. 1989). A single loop of similar extent has been detected south of 30 Dor in the LMC (Klein et al. 1993). Urbanik et al. (1993) modeled the distribution of polarized emission in M31 by a mixture of random and helically twisted magnetic fields.

Field lines emerging out of the plane are a common phenomenon among spiral galaxies (e.g. M. Krause et al. 1989a; Neininger et al. 1991). A single flux tube could be distinguished in M51 (Horellou et al. 1992) above an interarm region. The vertical field in the SW quadrant of NGC 6946 is of even larger extent and resembles a solar coronal hole (Beck 1991b). Streamers based in active regions in the plane have been observed in NGC 4631 (Golla \& Hummel, this volume), M82 (Reuter et al. 1992) and possibly also in NGC 253 (Carilli et al. 1992).

Highest polarized intensities of radio continuum emission are generally observed in interarm regions or outside the region of optical spiral arms, while polarization in spiral arms is very low (Figs. 7 and 8, see also IC 342 (M. Krause, this volume)). The total radio continuum emission is slightly enhanced at the inner edges of spiral arms, i.e. the average strength of the total field is strongest. The low degree of polarization there cannot be explained by Faraday depolarization; beam depolarization by unresolved field structures is the major effect. Star-forming processes seem to tangle the field lines. 


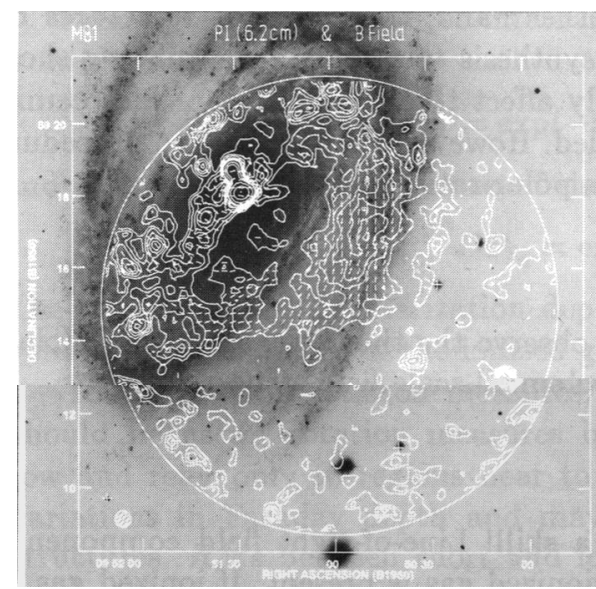

Fig. 7. Polarized emission and B-vectors from $\mathrm{M81}$ at $\lambda 6.2 \mathrm{~cm}$, observed with the VLA in its D configuration with $25^{\prime \prime}$ synthesized beam (Schoofs 1992).

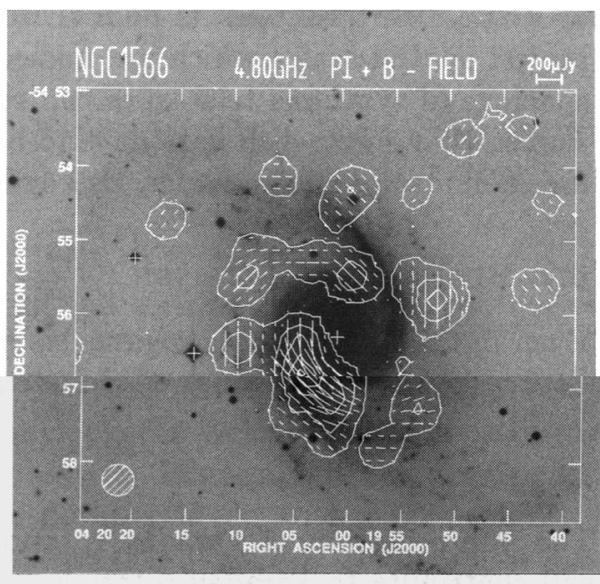

Fig. 8. Polarized emission and B-vectors from NGC 1566 at $\lambda 6.2 \mathrm{~cm}$, observed with the ATCA in its $375 \mathrm{~m}$ configuration with $26^{\prime \prime}$ synthesized beam (Beck, Haynes and Harnett, in prep.).

A qualitative model to explain field tangling has been proposed by Beck (1991b): Field lines are anchored in gas clouds. As the clouds enter a spiral arm, they are decelerated, and their number density, collision rate and turbulent velocity increase which gives rise to field tangling. In the interarm regions the gas clouds are accelerated, and the field lines are stretched out. If the distribution and motion of gas clouds is of such importance for the interstellar field, dynamo theory will have to account for it.

\section{Limitations and Problems of Radio Observations}

Radio polarization observations are difficult to obtain and even more difficult to interprete because interstellar magnetic fields exhibit a wealth of structures.

\subsection{INSTRUMENTAL EFFECTS}

Radio polarization observations impose highest requirements to the sensitivity and stability of the receiving system. Instrumental polarization has to be minimized. This problem is particularly critical for synthesis observations of extended objects like galaxies because instrumental polarization increases off the field center.

The VLA polarization map of M31 (Fig. 2) has been combined from 4 synthesized maps with different field centers without significant deviations in the overlap areas. Hence the VLA gives reliable results, at least in case of the absence of strong sources in the field.

Single dishes do not suffer from such effects because their instrumental polarization characteristics do not vary across the field. In case of strong sources, subtraction of instrumental polarization is possible. Unfortunately, the angular resolution 
of single-dish telescopes is poor. On the other hand, they still see structures on largest spatial scales which are missed by synthesis telescopes. The missing short spacings of synthesis telescopes can strongly affect the distribution of radio emission so that single-dish data have to be added. However, there is still no procedure for combination of single-dish and synthesis polarization data.

\subsection{Beam Depolarization}

Even in the Faraday-thin regime, we never observe the theoretical degree of polarization $\left(p_{0} \cong 74 \%\right)$ because of the unresolved small-scale field structures.

\subsection{Faraday Rotation}

The interpretation of Faraday rotation is a skill! Line-of-sight field components will contribute to Faraday rotation only if ionized gas is present. If ionized gas is restricted to the thin disk, the distribution of Faraday rotation gives information only about the field structure in the thin disk, which may be different from the field structure in the (more extended) synchrotron-emitting disk. This may explain the "wrong" phase of the $R M$ variation in NGC 6946 (Fig. 5).

Ionized gas in the thin disk is strongly clumped (HII regions) with a volume filling factor of only 5\% (Ehle \& Beck 1993) while the thick-disk gas has a much higher filling factor (e.g. Reynolds 1991). In NGC 6946 all data related to the ionized gas (Faraday rotation, Faraday depolarization and thermal emission measures) can be consistently explained by a thin-disk population of small HII regions (size $\cong$ $1 \mathrm{pc}$ ) with typically $\cong 5 \mathrm{~cm}^{-3}$ internal electron density. Hence Faraday rotation probably traces the average uniform magnetic field in the HII regions only. In M83 both thin and thick disk contribute to Faraday rotation (Neininger et al. 1993). Here the large-scale $R M$ variation reflects field properties of both components.

\subsection{Faraday Depolarization}

Varying field orientations along the line of sight give rise to dispersion in rotation measures (Faraday dispersion) which in turn leads to depolarization of the radio emission (Burn 1966). The field component with random orientations can be the field distorted by HII regions, stellar-wind driven bubbles, supernova remnants, Alfvén waves or instabilities.

Faraday dispersion means that Faraday rotation is no longer proportional to $\lambda^{2}$, or, in other words, the effective "Faraday depth" decreases with increasing wavelength. At long wavelengths, galaxies are no longer transparent to polarized radio waves. If Faraday dispersion is due to fluctuations in electron density, the variance $\sigma_{\mathrm{RM}}$ depends on size $d$ and filling factor $f$ of the turbulent cells, their internal electron density $n_{\mathrm{e}}$ and random field strength $B_{\mathrm{r}}$ (Burn 1966; Ehle \& Beck 1993):

$$
\sigma_{\mathrm{RM}}=\left(0.81 n_{\mathrm{e}} B_{\mathrm{r}} / \sqrt{3}\right)^{2} \cdot f \cdot d \cdot(L / \cos i)
$$

In the thin disk of HII regions $(L \cong 100 \mathrm{pc})$ we expect strong depolarization, while little dispersion is expected from diffuse ionized gas in the thick disk (Table V). 
Faraday dispersion leads to internal depolarization of the synchrotron emission from the same volume:

$$
D P_{1}=\exp \left(-\sigma_{\mathrm{RM}} \lambda^{4}\right) /\left(\sigma_{\mathrm{RM}} \lambda^{4}\right) .
$$

and to foreground depolarization of the background polarized emission:

$$
D P_{\mathrm{fg}}=\exp \left(-2 \sigma_{\mathrm{RM}} \lambda^{4}\right)
$$

The total observed depolarization depends on the distributions of the sources of polarized emission and of the ionized gas.

To obtain true rotation measures, only observations at wavelengths $\leqq 6 \mathrm{~cm}$ should be used. Rotation measures between longer wavelengths are mostly too low and refer only to regions near to the observer. Hence $R M$ variations reflect variations in Faraday depth and may have nothing to do with large-scale field structures. $R M$ s between short and long wavelengths (e.g. $2.8 / 20 \mathrm{~cm}$ ) often give no useful information because data from different regions are combined.

Even in case of completely uniform field, depolarization occurs due to differential Faraday rotation along the line of sight (Burn 1966):

$$
D P_{2}=\left|\sin \left(2 \cdot R M \cdot \lambda^{2}\right) /\left(2 \cdot R M \cdot \lambda^{2}\right)\right|
$$

where $R M$ is the internal rotation measure observed in the Faraday-thin regime. $D P_{2}=0$ occurs if $R M \cdot \lambda^{2}= \pm n \cdot \pi / 2$, accompanied by a $\pi / 2$-jump in $R M$. At $\lambda 20 \mathrm{~cm}$, for example, total depolarization occurs at $R M \cong 38 \mathrm{rad} \mathrm{m}^{-2}$ where $90^{\circ}$ Faraday rotation is reached. Higher $R M$ values than $\pi / 2 \lambda^{2}$ are difficult to detect because of strong depolarization. Higher internal Faraday rotation than $90^{\circ}$ can never be observed.

Differential Faraday rotation produces the total depolarization of the SW quadrant of NGC 6946 at $\lambda 20 \mathrm{~cm}$ (Beck 1991b; Ehle \& Beck, this volume) and some holes in the polarization maps of M51 at $\lambda 20 \mathrm{~cm}$ (Horellou et al. 1992). Such holes should not be misinterpreted as field minima.

Table $\mathrm{V}$ gives typical values for Faraday rotation and depolarization in galactic disks of thickness $L$, assuming strengths of $5 \mu \mathrm{G}$ for both random and uniform magnetic fields. $\left\langle n_{\mathrm{e}}\right\rangle$ is the average electron density $\left.\left(<n_{\mathrm{e}}\right\rangle=f \cdot n_{\mathrm{e}}\right) . R M$ is calculated for inclination $i=45^{\circ}, \Delta \chi$ is the corresponding Faraday rotation angle. $D P_{2}$ is the depolarization due to differential Faraday rotation, $D P_{1}$ that due to Faraday dispersion. Values for $D P_{1}$ and $D P_{2}$ in Table $\mathrm{V}$ refer to synchrotron emission from the thin or thick disk only. $D P_{\mathrm{fg}_{\mathrm{g}}}$ is the foreground depolarization of the emission from behind the thin disk. Emission from in front of the disks of ionized gas will not be depolarized. If we assume a synchrotron-emitting disk with $L_{\text {sync }} \cong 1 \mathrm{kpc}$, the total expected depolarization $D P_{\text {tot }}$ can be computed (Table V, last line).

At $\lambda \leq 6 \mathrm{~cm}$, Faraday depolarization is small even in opaque galaxies. At longer wavelength, Faraday depolarization depends on the ionized gas distribution. A thin disk of ionized gas can only depolarize about half of the synchrotron emission (as in NGC 6946, Ehle \& Beck 1993), while a thick disk of considerable electron density can fully depolarize the radio emission at $\lambda \cong 20 \mathrm{~cm}$, as has been observed in M83 (Neininger et al. 1993; Neininger et al., this volume). 
TABLE V

Typical expected Faraday rotation and depolarization in spiral galaxies (inclination $45^{\circ}$ )

\begin{tabular}{|c|c|c|c|c|}
\hline & \multicolumn{2}{|c|}{ Thermal thin disk } & \multicolumn{2}{|c|}{ Thermal thick disk } \\
\hline $\bar{L}$ & $100 \mathrm{pc}$ & & $1000 \mathrm{pc}$ & \\
\hline$\left\langle n_{e}\right\rangle$ & $0.3 \mathrm{~cm}^{-3}$ & & $0.03 \mathrm{~cm}^{-3}$ & \\
\hline & 0.05 & & 0.5 & \\
\hline & $1 \mathrm{pc}$ & & $50 \mathrm{pc}$ & \\
\hline $\begin{array}{l}R M_{\max } \\
\sigma_{\mathrm{RM}}\end{array}$ & $\begin{array}{l}61 \mathrm{rad} \mathrm{m}^{-2} \\
1400 \mathrm{rad}^{2} \mathrm{~m}^{-1}\end{array}$ & & $\begin{array}{l}61 \mathrm{rad} \mathrm{m}^{-2} \\
700 \mathrm{rad}^{2} \mathrm{~m}^{-4}\end{array}$ & \\
\hline & $(\lambda=6 \mathrm{~cm} \quad /$ & $\lambda=20 \mathrm{~cm})$ & $(\lambda=6 \mathrm{~cm} \quad /$ & $\lambda=20 \mathrm{~cm})$ \\
\hline$\Delta \chi$ & $13^{\circ}$ & $\left(90^{\circ}\right)$ & $13^{\circ}$ & $\left(90^{\circ}\right)$ \\
\hline$D P_{2}$ & 0.97 & 0 & 0.97 & 0 \\
\hline$D P_{1}$ & 0.99 & 0.40 & 0.99 & 0.60 \\
\hline$D P_{f_{g}}$ & 0.96 & 0.01 & - & - \\
\hline$D P_{\text {tot }}$ & 0.98 & 0.46 & 0.96 & $\mathbf{0}$ \\
\hline
\end{tabular}

\section{Needs for Future Observations}

To observe small-scale field structures, single-dish telescopes should be operated at mm wavelengths. A multi-beam $\lambda 9 \mathrm{~mm}$ receiving system for the Effelsberg telescope (giving $25^{\prime \prime}$ resolution) is under construction. Synthesis telescopes should be used in the Faraday-thin regime, e.g. at $\lambda \leq 6 \mathrm{~cm}$. To cover the whole field of a galaxy, mosaicing is required, to be combined with single-dish observations at a similar wavelength to add the missing large-scale emission.

Faraday rotation is best studied in the Faraday-thin regime, e.g. between $\lambda 2.8 \mathrm{~cm}$ (Effelsberg) and $\lambda 6.2 \mathrm{~cm}$ (WSRT, VLA or ATCA). In galaxies with little Faraday rotation a longer wavelength $(11-13 \mathrm{~cm})$ is necessary to obtain sufficient accuracy. Only the Effelsberg, Parkes and ATCA telescopes operate at such wavelengths.

Studies of Faraday depolarization should work in the transition regime between large and small Faraday depths. For more transparent galaxies $\lambda 20 \mathrm{~cm}$ is adequate, but for opaque galaxies like M83 a shorter wavelength is preferable. Several frequencies in the transition regime between small and large Faraday depth would give access to different layers in the disk or halo of spiral galaxies.

\section{References}

Beck R.: 1982, $A \& A$ 106, 121

Beck R., Loiseau N., Hummel E., Berkhuijsen E.M., Gräve R., Wielebinski R.: 1982, A\&A 222 , 58

Beck R.: 1991a, in H. Bloemen, ed(s)., The Interstellar Disk-Halo Connection in Galaxies, Kluwer: Dordrecht, p. 267

Beck R.: 1991b, A\&A 251, 15

Brandenburg A., Donner K.J., Moss D., Shukurov A., Sokoloff D.D., Tuominen I.: 1992, A\&A 259,453 
Brandenburg A., Donner K.J., Moss D., Shukurov A., Sokoloff D.D., Tuominen I.: 1993, A\&A (submitted)

Buczilowski U.R., Beck R.: 1991, $A \varangle A$ 241, 47

Burn B.J.: 1966, MNRAS 133, 67

Carilli C.L., Holdaway M.A., Ho P.T.P., De Pree C.G.: 1992, ApJ 399, L59

Chiba M., Tosa M.: 1989, MNRAS 238, 621

Chiba M., Tosa M.: 1990, MNRAS 244, 714

Donner K.J., Brandenburg A.: 1990, A\&A 240, 289

Ehle M., Beck R.: 1993, $A \& A$ (in press)

Elstner D., Meinel R., Beck R.: 1992, A\&AS 94, 587

Gräve R., Beck R.: 1988, $A \& A$ 192, 66

Hanasz M., Lesch H., Krause M.: 1991, $A \& A$ 243, 381

Horellou C., Beck R., Klein U.: 1990, in R. Beck, P.P. Kronberg, R. Wielebinski, ed(s)., Galactic and Intergalactic Magnetic Fields, Kluwer: Dordrecht, p. 211

Horellou C., Beck R., Berkhuijsen E.M., Krause M., Klein U.: 1992, A\&A 265, 417

Hummel E., Beck R., Dahlem M.: 1991, A\&A 248, 23

Klein U., Urbanik M., Beck R., Wielebinski R.: 1983, $A$ \&A 127, 177

Klein U., Haynes R.F., Wielebinski R., Meinert D.: 1993, $A \& A$ (in press)

Krasheninnikova Y., Ruzmaikin A., Sokoloff D., Shukurov A.: 1989, A\&A 213, 19

Krause M.: 1990, in R. Beck, P.P. Kronberg, R. Wielebinski, ed(s)., Galactic and Intergalactic Magnetic Fields, Kluwer: Dordrecht, p. 187

Krause M., Hummel E., Beck R.: 1989a, $A \& A$ 217, 4

Krause M., Beck R., Hummel E.: 1989b, $A \& A$ 217, 17

Kronberg P.P., Perry J.J., Zukowski E.L.H.: 1992, ApJ 387, 528

Lesch H., Harnett J.I.: 1993, $A \& A$ (in press)

Moss D., Brandenburg A., Donner K.J., Thomasson M.: 1993, ApJ (in press)

Neininger N.: 1992a, $A \& A$ 263, 30

Neininger N.: 1992b, Ph.D. thesis, Bonn University

Neininger N., Klein U., Beck R., Wielebinski R.: 1991, Nature 352, 781

Neininger N., Beck R., Sukumar S., Allen R.J.: 1993, A\&A (submitted)

Perry J.J., Watson A.M., Kronberg P.P.: 1993, ApJ (in press)

Reuter H.-P., Krause M., Wielebinski R., Lesch H.: 1991, $A \& A$ 248, 12

Reuter H.-P., Klein U., Lesch H., Wielebinski R., Kronberg P.P.: 1992, A\&A 256, 10

Reynolds R.J.: 1991, in H. Bloemen, ed(s)., The Interstellar Disk-Halo Connection in Galaxies, Kluwer: Dordrecht, p. 67

Rüdiger G., Elstner D., Schultz M.: 1993, A\&A (in press)

Ruzmaikin A., Sokoloff D., Shukurov A., Beck R.: 1990, A\&A 230, 284

Schoofs S.: 1992, Diplomarbeit, Bonn University

Sofue Y., Beck R.: 1987, PASJ 39, 541

Sofue Y., Klein U., Beck R., Wielebinski R.: 1985, $A \& A$ 144, 257

Sokoloff D., Shukurov A., Krause M.: 1992, $A \& A$ 264, 396

Spencer S.J., Cram L.E.: 1992, ApJ 400, 484

Sukumar S., Allen R.J.: 1991, ApJ 382, 100

Urbanik M., Otmianowska-Mazur K., Beck R.: 1993, A\&A (submitted)

Vallée J.P.: 1990, ApJ 361, 419

Wolfe A.M., Lanzetta K.M., Oren A.L.: 1992, ApJ 388, 17 